\title{
Identification of heat exchangers according to the criterion of energy efficiency
}

\author{
Vladimir Velichkin ${ }^{1 *}$ and Vladimir Zavyalov ${ }^{1}$ \\ ${ }^{1}$ Department of Scientific, National Research Moscow State University of Civil \\ Engineering, Moscow, Russia
}

\begin{abstract}
The article presents the results of the analysis of the characteristics of heat exchangers methods for determining their mathematical models. The necessity of the availability of the mathematical model during the synthesis of automatic control systems with desired properties. The method of identification of the thermal control object by the testing control action is proposed. Since technological control objects always be an energy of interaction the energy efficiency criterion applied for automatic formation of the control action. Also the analytical selfadjusting system with a reference model in the form of an integrating link was applied. From the analytical researches it follows that the movement of the system along the optimal trajectory occurs at a constant speed and does not depend on the properties of the control object, and the optimal control depends on the properties of the control object, time, and technological requirements. It is shown that mathematical models of heat exchangers of the first and second orders are determined quite simply. The accuracy of the mathematical model parameters is limited only by the accuracy of the experimental data. The quality of control systems with desired properties, synthesized by experimental, and accurate models are virtually indistinguishable.
\end{abstract}

\section{Introduction}

The flow of heat in process equipment does not have a kinetic component. This suggests that dynamic processes in heat exchangers (TOA) can be described with the help of exponential dependences. Also, many TOA is inertial objects and not only to respond to rapidly changing interference. From the above, we can conclude that the thermal processes in the TOA can be characterized by models described by deterministic linear or nonlinear differential equations.

Unfortunately, the values of the coefficients of differential equations are not always obtained by calculation, and their definition requires experimental studies. This is explained by the fact that the parameters of the heat exchange process depend on some uncertain process parameters. For example, the heat exchange coefficient depends on the properties of the heat exchange surface, the properties of the heat exchange media, the relative velocity of the heat exchange media, the temperature, pressure and so on.

\footnotetext{
* Corresponding author: VelichkinVA@gic.mgsu.ru
} 
The structure of the model is determined by the design of the TOA and the scheme of thermal flows in it. The system of describing equations is generally composed of thermal balances between the TOA elements. Thus values of coefficients of the describing equations are defined in the course of identification of the investigated object as the control object.

Identification is a necessary condition for the creation of an automatic control system (ACS) with specified properties.

Currently, there are many works [1], [3], [6] on the identification of the control object (OU) with the help of standard input control and disturbing influences. The particularity of this article is that non-standard impacts are applied to the input of an OU. These impacts are determined according to the optimality criterion of energy efficiency. This problem was solved for the first time.

The process of choosing the testing effects is subjective, and the adequacy of the model to the object is achieved with various success. Here it should be noted that the OU model is only a representation of the real OU in the researcher's imagination and can never coincide with the properties of the real OU. It remains only to strive for the maximum proximity of the properties of OU and the model suitable for its further use.

Too detailed models of OU can lead to computational difficulties and computational errors, and too simplified do not allow to achieve the desired result in the synthesis of ACS with specified properties. Model errors of $5-10 \%$ can be compensated by adjusting the parameters of the control device. When you change the properties of the OU process in the normal functioning of the ACS tuning parameters of the control devices must be performed with the built-in ACS system identification (SI).

\section{Methodology}

Since technological control objects are always the energy of interaction for the formation of the impact testing, it is advisable to choose the criterion of energy efficiency [1]. This criterion it was used for optimization of ACS by thermal objects [2].

$$
J=\int_{0}^{T_{u}}\left(X^{T} A^{T} A X+2 X^{T} A^{T} B U+U^{T} B^{T} B U\right) d \tau
$$

where $X=$ the state vector of the control object, $A=$ the matrix of the dynamic properties of the control object, $B=$ influence matrix of control actions on the control object, $U=$ control effects on the control object, $T=$ the sign of the matrix transposition and time-management, $T_{U}=$ the length of the interval control, $\tau=$ current time.

Solution of the optimal control problem by this criterion for objects whose model is presented in matrix form.

$$
X=A X+B U
$$

It was obtained analytically and has the form:

$$
\begin{gathered}
U^{0}(\tau)=B^{-1} \cdot\left[-A \cdot X^{0}+\frac{X_{T}-X_{0}}{T_{U}}\right] \\
X_{0}=X_{0}+\frac{X_{T}-X_{0}}{T_{U}} \cdot \tau
\end{gathered}
$$


where $U^{0}=$ optimal control, $B^{-1}=$ inverse matrix of influence of control actions on the control object, $A=$ the matrix of the dynamic properties of the control object, $X_{0}=$ initial state of the control object,

$$
X_{T}=\text { final state of the control object, } \frac{X_{T}-X_{0}}{T_{U}} \quad=\text { technological requirement }
$$

From expressions (3) and (4) it follows that the movement of the system along the optimal trajectory occurs at a constant speed and does not depend on the properties of the control object, and the optimal control depends on the properties of the control object, time, and technological requirements.

\section{Results and discussion}

If we ensure the movement of the object at a constant speed, then the change in time of the testing control action, depending on the unknown properties of the $\mathrm{OU}$, it is possible to determine the parameters of the OU experimentally. For realization of the set task it is expedient to use analytical self-adjusting system of automatic control with reference model [3] which scheme is presented in Fig. 1.

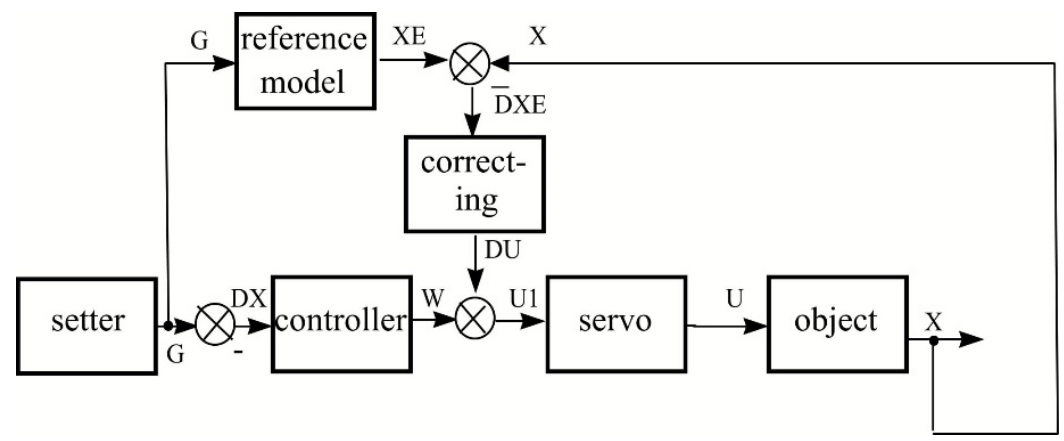

Fig. 1. Scheme of analytical self-adjusting automatic control system with reference model without basic feedback.

In the presented scheme (Fig. 1) the main feedback during the identification period is excluded and the following elements of the ACS are left:

setter $-G(p)=\frac{1}{p}$

reference model $-W_{E}(p)=\frac{C}{p}$

$C=\frac{X_{T}-X_{0}}{T_{U}}-$ rate of change of the output variable of the reference model

controller $-W_{R}(p)=\frac{K I}{p}$

$K I$ - the transmission coefficient of the integral controller

corrective link $-W_{K}(p)=K$

Executive mechanism with regulation. on the (IM - RO) $-W_{I R}(p)=K_{0}$

chamber (heat exchanger) $-W_{0}(p)=\frac{R_{0}(p)}{Q_{0}(p)}$ 
$R_{0}(p)$ - the polynomial of the numerator

$Q_{0}(p)$ - the polynomial of the denominator

At zero initial conditions $X_{0}=0$, the expressions (3) and (4) take the following form

$$
\begin{gathered}
U^{0}(\tau)=B^{-1} \cdot\left[-A \cdot X^{0}+\frac{X_{T}}{T_{U}}\right]=B^{-1} \cdot[I-A \cdot \tau] \cdot \frac{X_{T}}{T_{U}} \\
X^{0}(\tau)=\frac{X_{T}}{T_{U}} \cdot \tau, C=\frac{X_{T}}{T_{U}}
\end{gathered}
$$

$I=$ identity matrix

or, subject to (7), expression (5) and (6) take the form of

$$
\begin{gathered}
U^{0}(\tau)=B^{-1} \cdot[I-A \cdot \tau] \cdot C \\
X^{0}(\tau)=C \cdot \tau .
\end{gathered}
$$

The value $C$ is chosen at identification such that the variables $\mathrm{X}^{0}$ and $\mathrm{U}^{0}-$ do not exceed the permissible values.

Example 1. Let the object be characterized by a differential equation

$$
\frac{d X(\tau)}{d \tau}=a \cdot X(\tau)+b \cdot U(\tau) \text {. }
$$

Then under $X(\tau)=C \cdot \tau, U(\tau)=\frac{C}{b} \cdot[1-a \tau]$.

At $\tau=0 \rightarrow U(0)=\frac{C}{b}$, therefore, $b=\frac{C}{U(0)}$.

At $\tau=T_{U} U\left(T_{U}\right)=U(0)\left[1-a T_{U}\right]$, therefore $a=-\frac{U\left(T_{U}\right)-U(0)}{U(0) \cdot T_{U}}$.

At known values from the experiment $U\left(T_{U}\right), U(0)$ and $T_{U}$ parameter values $a$ and $b$ are easily calculated.

At $U(0)=1, U(1)=2, T_{U}=1, X_{T}=1$ и $C=1 \rightarrow a=-1, b=1$.

Example 2. Let the object be characterized by a differential equation

$$
\frac{d^{2} X(\tau)}{d \tau^{2}}+a_{1} \frac{d X(\tau)}{d \tau}+a_{2} X(\tau)=b U(\tau)
$$

Then under $X(\tau)=C \cdot \tau$ and $\frac{d X(\tau)}{d \tau}=C$ Eq. 10 takes the form

$$
a_{1} \cdot C+a_{2} \cdot C \cdot \tau=b U(\tau) \text { or } U(\tau)=\frac{C}{b}\left[a_{1}+a_{2} \cdot \tau\right]
$$

If we take $C=b$, then Eq. 11 takes the form

$$
U(\tau)=\left[a_{1}+a_{2} \cdot \tau\right]
$$


From Eq. 12 follows:

$$
\begin{aligned}
& U(0)=a_{1}, a_{1}=U(0), U(1)=\left[a_{1}+a_{2}\right]=\left[U(0)+a_{2}\right], a_{2}=U(1)-U(0), \\
& b=C=\frac{U(1)-U(0)}{\Delta \tau}
\end{aligned}
$$

On the basis of the experimental and the above dependencies, the coefficients of the Eq. 10 characterizing the properties of the control object can be determined. On experimental data $U(0)=1.5, U(1)=2.0, \Delta=1.0$. Then

$$
\begin{gathered}
\quad b=C=\frac{U(1)-U(0)}{\Delta \tau}=\frac{2-1.5}{1}=0.5 \\
a_{1}=U(0)=1.5, a_{2}=U(1)-U(0)=2-1.5=0.5
\end{gathered}
$$

The results suggest that the proposed method of identification has a significant accuracy, which is limited by the accuracy of the experimental data.

Example 3. In example 2, the control object is a product of two aperiodic links of the first order. In practice, it is often necessary to represent a model of a control object as a sum of two aperiodic units. In this case, the object is characterized by a second-order aperiodic link of the following form.

$$
W(p)=\frac{b \times(T p+1)}{b_{0} p^{2}+b_{1} p+1}=\frac{0.25 \times(p+1)}{0.64 p^{2}+2.1 p+1}
$$

Then the testing control in the operator form has the form.

$$
U(p)=\frac{2.56 p^{2}+8.4 p+4}{p^{2} \cdot(p+1)}
$$

The original test control (14) for [4] is characterized by the following dependence

$$
U(\tau)=\frac{1}{b} \cdot\left[C_{1}+C_{2} \cdot e^{-\alpha \tau}+\tau\right]=4 \cdot C_{1}+4 \cdot C_{2} \cdot e^{-\alpha \tau}
$$

where $C_{1}=b_{1}-\frac{1}{\alpha}, C_{2}=b_{0} \cdot \alpha+\left(\frac{1}{\alpha}-b_{1}\right), \alpha=\frac{1}{T}$

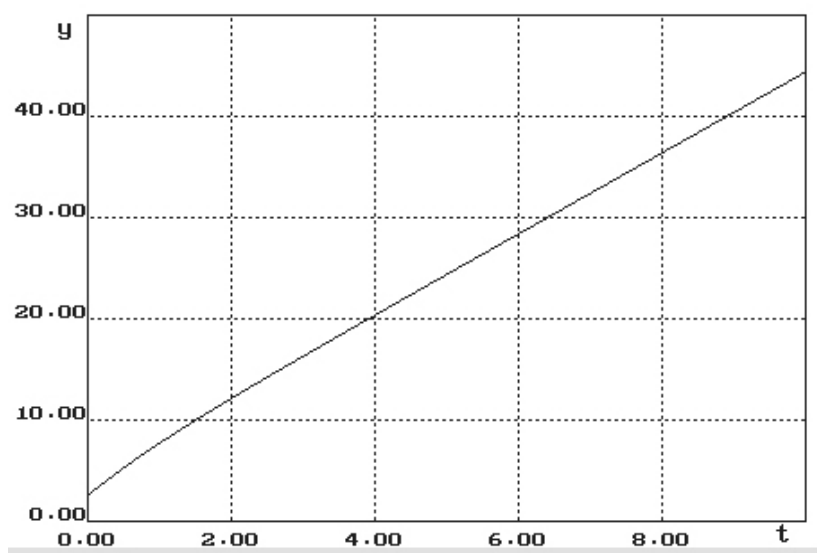

Fig. 2. The graph of the function (15) test control. 
Values can be obtained from experimental data (Fig. 2 and Table 1) and calculated by dependencies $U(\tau)=\frac{1}{b}\left[C_{1}+\tau+C_{2} \cdot e^{-\alpha \tau}\right]$ and $\frac{1}{b}=\frac{U(i)-U(i-1)}{\Delta \tau} \approx \frac{X_{T}}{T_{U}}$.

Table. 1 The table of the function (15) test control.

\begin{tabular}{|c|c|c|c|c|c|c|}
\hline$\tau$ & $\mathbf{0}$ & $\mathbf{1}$ & $\mathbf{2}$ & $\mathbf{3}$ & $\mathbf{4}$ & $\mathbf{5}$ \\
\hline $\mathrm{U}(\tau)$ & 2.57 & 7.75 & 12.16 & 16.32 & 20.34 & 24.39 \\
\hline$\Delta \mathrm{U}(\tau)$ & & 5.18 & 4.41 & 4.16 & 4.02 & 4.05 \\
\hline$\tau$ & 6 & 7 & 8 & 9 & 10 & 11 \\
\hline $\mathrm{U}(\tau)$ & 28.41 & 32.41 & 36.41 & 40.41 & 44.41 & 48.41 \\
\hline$\Delta \mathrm{U}(\tau)$ & 4.02 & 4.0 & 40 & 4.0 & 4.0 & 4.0 \\
\hline
\end{tabular}

Based on the obtained experimental data:

$$
\frac{1}{b}=\frac{U(11)-U(10)}{\Delta \tau}=\frac{44.41-48.41}{1}=4.0
$$

At $\tau=10$

$$
\begin{gathered}
U(10)=C_{1}+40+C_{2} \cdot e^{-10 \alpha}=44.41, C_{2} \cdot e^{-10 \alpha} \cong 0 \\
4 \cdot C_{1}=44.41-40=4.41, C_{1} \cong 1.1
\end{gathered}
$$

At $\tau=0$

At $\tau=1$

$$
\begin{gathered}
U(10)=4 C_{1}+4 C_{2}=2.57,4 C_{2}=2.57-4.41=-1.84 \\
C_{2}=-\frac{1.84}{4}=-0, .46
\end{gathered}
$$

$$
\begin{gathered}
U(1)=4.41+4-1.84 e^{-\alpha}=7.74 \\
e^{-\alpha}=\frac{8.41-7.74}{1.84}=0.364 \\
-\alpha=\ln 0.364=-1.01
\end{gathered}
$$

Thus, according to the results of calculations:

$b=0.25(0.25), T=\frac{1}{\alpha}=\frac{1}{1.01}=0.99$ (1.0)

$b_{1}=C_{1}+T=1.1+0.99=2.09(2.1)$

$b_{0}=\frac{C_{2}+b_{1}-T}{\alpha}=\frac{-0.46+2.09-0.99}{1.01}=0.634(0.64)$

$W(p)=\frac{b \times(T p+1)}{b_{0} p^{2}+b_{1} p+1}=\frac{0.25 \times(p+1)}{0.64 p^{2}+2.1 p+1} \cong \frac{0.25 \times(0.99 p+1)}{0.634 p^{2}+2.09 p+1}$

Errors in the calculation of transfer function coefficients can be explained by errors in the experimental data. The obtained identification results can be applied for the synthesis of traditional automatic control systems with desired properties [5] with a microprocessor control device, MPACS.

Transitional functions of the original and calculated MPACS in operator and time forms are characterized by the following expressions. 


$$
\begin{gathered}
H(p)_{3}=\frac{1}{p^{2}+p} \text { and } H(\tau)_{3}=1-e^{-\tau} \\
H(p)_{3}=\frac{0.634 p^{3}+2.724 p^{2}+3.09 p+1}{0.6336 p^{3}+3.363 p^{4}+5.814 p^{3}+4.09 p^{2}+p}
\end{gathered}
$$

Transition functions (16) and (17) for calculations on a PC are practically the same.

\section{Conclusions}

A combination of exponential dependencies can characterize thermal processes in technological devices with sufficient accuracy for practice. The energy efficiency criterion used for identification for the first time allows forming an optimal test control signal. To keep the movement of the control object on the optimal trajectory, it is advisable to use a self-adjusting system with a reference model in the form of an ideal integrating link. The studies have shown that the coefficients of the mathematical model of the control object can be determined with sufficient accuracy by the experimental testing control action. The use of original and experimental control models for the synthesis of ACS with specified properties gave virtually identical results.

\section{References}

1. V.A. Zavyalov, Theory and practice of optimization of operational management of heat treatment of concrete building products. (Thesis MGSU, Moscow, 2001)

2. V.A. Zavyalov, Building Material XXI Century (2001)

3. V.V. Solodovnikov, L.S. Shramko, Calculation and design of analytical self-adjusting systems with reference models. (Moscow, 1972)

4. I.M. Makarov, B.M. Mensky, Linear automatic systems (Elements of theory, calculation methods and reference material) (Mashinostroenie, 1982)

5. V.A. Zavialov, V.A. Velichkin, Mechanization of Construction, 2 (2012)

6. A.G. Strizhnev, A.V. Markov, Identification of control object by the transitional characteristic of a closed system (BSUIR, Belarus, 2012) 